\title{
Exercised-Induced Ischemic Colitis in a Recreational Athlete Previously Attributed to Inflammatory Bowel Disease
}

\author{
Michael P. Lukela, ${ }^{\mathrm{a}, \mathrm{b}}$, William D. Chey
}

\begin{abstract}
Ischemic colitis traditionally presents in older patients, many whom have underlying atherosclerotic disease. Although symptoms may be transient and resolve spontaneously, serious complications may develop including stricture formation, bowel perforation, sepsis, or death. Recently, otherwise healthy athletes have been reported to develop ischemic colitis during or following strenuous, prolonged exercise. Here we describe a unique case in which a recreational athlete previously diagnosed with Crohn's disease ten years prior presented with diarrhea and hematochezia attributed to an acute flare of her inflammatory bowel disease. Thorough review of her history, physical examination, laboratory, radiographic, and endoscopic evaluations revealed that her presenting symptoms were secondary to exercise-induced ischemic colitis. Moreover, her evaluation suggested that her prior diagnosis of Crohn's disease was incorrect and that her previous Crohn's flares were likely secondary to intermittent bouts of ischemic colitis. This case highlights the importance of recognizing the diagnosis of ischemic colitis in an atypical host, but one in which the disease prevalence is likely to increase as more individuals participate in recreational endurance activities.
\end{abstract}

Keywords: Ischemic colitis; Mesenteric ischemia; Gastrointestinal bleeding; Marathon running; Case report

\section{Introduction}

The prevalence of recreational endurance athletes is increasing rapidly. Between 2007 and 2008 the number of marathon

\footnotetext{
Manuscript accepted for publication August 13, 2012

${ }^{a}$ Department of Internal Medicine, University of Michigan Hospitals, USA

${ }^{\mathrm{b}}$ Department of Pediatrics, University of Michigan Hospitals, USA

${ }^{\mathrm{c} C}$ Corresponding author: Michael P. Lukela, 3116 Taubman Center, SPC

5368, 1500 E. Medical Center Drive, USA.

Email:mlukela@umich.edu
}

doi: http://dx.doi.org/10.4021/jmc839w runners in the United States rose $3.7 \%$ to over 400,000 participants. Ischemic colitis traditionally presents in older patients, many whom have underlying atherosclerotic disease. Although symptoms may be transient and resolve spontaneously, serious complications may develop including stricture formation, bowel perforation, sepsis, or death. Recently, otherwise healthy athletes have been reported to develop ischemic colitis during or following strenuous, prolonged exercise. Here we describe a unique case in which a recreational athlete previously diagnosed with Crohn's disease ten years prior presented with diarrhea and hematochezia attributed to an acute flare of her inflammatory bowel disease. Thorough review of her history, physical examination, laboratory, radiographic, and endoscopic evaluations revealed that her presenting symptoms were secondary to exercise-induced ischemic colitis. Moreover, her evaluation suggested that her prior diagnosis of Crohn's disease was incorrect and that her previous Crohn's flares were likely secondary to intermittent bouts of ischemic colitis. This case highlights the importance of recognizing the diagnosis of ischemic colitis in an atypical host, but one in which the disease prevalence is likely to increase as more individuals participate in recreational endurance sports.

\section{Case Report}

A 29-year-old woman with a history of Crohn's disease presented with two days of diarrhea and hematochezia. She was diagnosed approximately ten years prior by colonoscopy and received mesalamine for one month, but discontinued this medication secondary to cost. Since her diagnosis, she reported recurrent abdominal cramping and non-bloody diarrhea recurring once to twice per month with episodes resolving spontaneously in less than 24 hours. Her symptoms were not associated with her menses and usually occurred following strenuous activity. During the six months prior to presentation, she recalled two episodes of severe abdominal cramping and diarrhea following strenuous running, although neither was associated with hematochezia.

Two days prior to admission, she developed severe lower abdominal cramping after running two miles. The quality of her pain was similar to prior episodes she associated with 


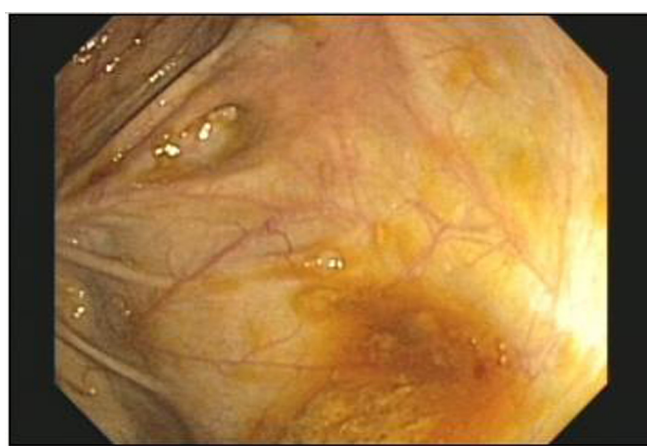

Figure 1. Colonic Ulcer. Image taken during colonoscopy showed a solitary, 20-millimeter ulcer located at the splenic flexure. The remainder of the colonic mucosa was normal to visual inspection. Pathology obtained following cold forceps biopsy at the ulcer bed was consistent with ischemic colonic mucosa. Additional random colonic biopsies were normal.

her Crohn's flares. She subsequently had five episodes of diarrhea consisting of loose, brown stool with hematochezia. She noted nausea, but denied emesis or fever. Her history was negative for known sick contacts, recent travel, antibiotics, non-steroidal anti-inflammatories (NSAIDs), contact with animals, or exposure to contaminated foods. Her review of systems was negative for oral ulcers, arthralgias, unexplained fevers, unintentional weight loss, or rash. Her past medical history was remarkable for depression. Her only medications were Ethinyl Estradiol/Levonorgestrel (Seasonale) and Fluoxetine. Family history revealed a 2nd cousin with inflammatory bowel disease, but she had no immediate family members with underlying gastrointestinal or autoimmune disorders. She rarely drank alcohol and was a lifelong non-smoker.

On exam she was well nourished and appeared mildly uncomfortable. She was afebrile with a heart rate of 65 beats per minute and blood pressure of 139/84 mmHg. Her abdomen was scaphoid, soft, and non-distended with normal active bowel sounds. She did not have hepatosplenomegaly or masses. She had tenderness in the periumbilical region and left mid-abdomen to deep palpation without guarding or rebound. Rectal examination revealed brown, guaiac positive stool; no hemorrhoids were noted. The remainder of her examination was unremarkable.

Laboratory testing showed 6,900 white blood cells (WBCs) $/ \mathrm{mm}^{3}$ with $63 \%$ neutrophils and $25 \%$ lymphocytes. Her hemoglobin was $13.3 \mathrm{~g} / \mathrm{dL}$. Erythrocyte sedimentation rate and C-reactive protein were $10 \mathrm{~mm} / \mathrm{Hr}$ and $0.4 \mathrm{mg} / \mathrm{dL}$, respectively. Electrolytes, renal function, liver function tests, amylase, lipase, and iron studies were normal. Fecal leukocytes, stool culture, examination for ova and parasites, and assay for Clostridium difficile toxin were negative. Computed tomography of the abdomen showed mild thickening of a small segment of the descending colon without evidence of perforation. She underwent colonoscopy that revealed a soli- tary twenty-millimeter ulcer at the splenic flexure without stigmata of recent or active hemorrhage (Fig. 1). Biopsies of the terminal ileum showed a prominent lymphoid follicle; biopsies at the splenic flexure were consistent with ischemic colitis. The patient was treated supportively with bowel rest and intravenous fluid hydration. Her symptoms resolved by the second hospital day. Upon discharge, she was counseled to carefully monitor her hydration and to increase fluid intake when engaging in physical activity. Two months following hospitalization, she remained asymptomatic and had returned to her baseline level of activity.

\section{Discussion}

Acute ischemic colitis infrequently presents in patients prior to the 5th decade of life with an estimated incidence of 15.6 cases per 100,000 patient years [1]. Associated co-morbidities that increase the risk of developing this condition include hypertension, chronic obstructive pulmonary disease, atrial fibrillation, congestive heart failure, and diabetes [2]. It is not clear whether males or females are more commonly affected as different studies report male [3], female [1], or equal gender predominance [4].

Gastrointestinal symptoms including anorexia, nausea, emesis, abdominal pain, diarrhea, and/or blood in the stool are common in endurance athletes during or following strenuous, prolonged physical activity [4-7]. In addition, several studies have demonstrated an increased incidence of occult gastrointestinal (GI) blood loss among athletes [8-10]. Gross GI blood loss following exercise is less common, although one study reported $16 \%$ of runners experienced bloody diarrhea on at least one occasion following strenuous exertion [6]. Development of ischemic colitis in this population is rare, however [7, 10, 11-14]. Several hypotheses to explain this phenomenon have be proposed. Mesenteric blood flow may decrease by $80 \%$ following strenuous exercise [15]. Additional physiologic changes precipitated by strenuous activity such as enhanced sympathetic tone, hypovolemia, hyperthermia, alterations in blood viscosity, and/or accumulation of vasoactive metabolites have been postulated to create low flow states predisposing susceptible individuals to develop ischemic colitis $[4,15]$. Associations with NSAIDs or oral contraceptives (OCPs) have also been described, but have not proved causal $[16,17]$.

Our case was unique in several respects. First, the patient was previously diagnosed with Crohn's disease. At the time of her initial diagnosis ten years prior, she noted intermittent abdominal pain and non-bloody diarrhea, but did not experience other symptoms such as weight loss, anorexia, or fatigue which is present in 50-70\% of patients with Crohn's disease [18]. Moreover, she did not have extra-intestinal manifestations such as erythema nodosum, oral ulceration, or ocular involvement which has been described in $5-15 \%$ 
of Crohn's patients [18]. The finding of lymphoid aggregates on her biopsy during her hospital admission did not demonstrate crypt distortion/atrophy; the villiform surface appearance suggests that this finding represents a normal variant rather than underlying pathology related to Crohn's disease [19]. Based on the patient's clinical history, laboratory studies, radiographic studies, endoscopy, and biopsy results her prior and presenting symptoms were not consistent with the diagnosis of inflammatory bowel disease. Second, our patient was a well-conditioned athlete who developed severe symptoms during a low-level of activity. Case reports have described ischemic colitis in both well-trained professional athletes and relatively unconditioned amateur athletes $[7,11$, 12, 14]. These reports, however, primarily described symptom onset during or following strenuous effort when athletes operated near their peak physiologic abilities, which was not the case for our patient. Although the patient had been taking OCPs for approximately five years, her symptoms at the time of her initial diagnosis ten years prior occurred in the absence of this medication. Careful review of her clinical history, exposures, laboratory studies were unable to identify additional contributing factors to explain her symptoms. This case highlights several important points. Abdominal pain, diarrhea, and hematochezia are common presenting symptoms. The development of exercise-induced ischemic colitis, however, is rare [7, 17]. Although the majority of cases in the literature describing exercise-induced ischemic colitis were managed conservatively, severe complications such as bowel perforation may develop and could be easily missed if the correct diagnosis is not entertained [12]. Misdiagnosis, too, can have longstanding consequences. In our case the patient was initially misdiagnosed with Crohn's disease. If her treatment remained focused on treating her for inflammatory bowel disease it is likely she would have received more intensive therapies such as corticosteroids or immune modulating agents and therefore been subject to their attendant side-effects and long-term health consequences. Moreover, the underlying condition driving her symptoms would not have been appropriately addressed.

Finally, it is important to recognize that the prevalence of recreational endurance athletes is increasing rapidly. Between 2007 and 2008 the number of marathon runners in the United States rose $3.7 \%$ to over 400,000 participants and future interest in endurance sports is likely to continue during the coming years [20]. As such, this case highlights the importance of recognizing the diagnosis of ischemic colitis in an atypical host, but one in which the disease prevalence is likely to increase as more individuals participate in recreational endurance activities.

\section{Authors Contributions}

Dr. Lukela identified the case described, wrote the draft of the manuscript, and revised the manuscript into its final form. Dr. Chey reviewed the drafting of the article, providing revisions for important intellectual content. Both authors fulfill the authorship criteria required by the Journal.

\section{Conflicts of Interest}

To the authors' knowledge there are no relevant conflicts of interest to report.

\section{References}

1. Longstreth GF, Yao JF. Epidemiology, clinical features, high-risk factors, and outcome of acute large bowel ischemia. Clin Gastroenterol Hepatol. 2009;7(10):10751080 e1071-1072; quiz 1023.

2. Longstreth GF, Yao JF. Diseases and drugs that increase risk of acute large bowel ischemia. Clin Gastroenterol Hepatol. 2010;8(1):49-54.

3. Longo WE, Ballantyne GH, Gusberg RJ. Ischemic colitis: patterns and prognosis. Dis Colon Rectum. 1992;35(8):726-730.

4. Scharff JR, Longo WE, Vartanian SM, Jacobs DL, Bahadursingh AN, Kaminski DL. Ischemic colitis: spectrum of disease and outcome. Surgery. 2003;134(4):624-629; discussion 629-630.

5. Peters HP, Bos M, Seebregts L, Akkermans LM, van Berge Henegouwen GP, Bol E, Mosterd WL, et al. Gastrointestinal symptoms in long-distance runners, cyclists, and triathletes: prevalence, medication, and etiology. Am J Gastroenterol. 1999;94(6):1570-1581.

6. Sullivan SN, Wong C. Runners' diarrhea. Different patterns and associated factors. J Clin Gastroenterol. 1992;14(2):101-104.

7. Sanchez LD, Tracy JA, Berkoff D, Pedrosa I. Ischemic colitis in marathon runners: a case-based review. J Emerg Med. 2006;30(3):321-326.

8. McMahon LF, Jr., Ryan MJ, Larson D, Fisher RL. Occult gastrointestinal blood loss in marathon runners. Ann Intern Med. 1984;100(6):846-847.

9. Baska RS, Moses FM, Graeber G, Kearney G. Gastrointestinal bleeding during an ultramarathon. Dig Dis Sci. 1990;35(2):276-279.

10. Choi SC, Choi SJ, Kim JA, Kim TH, Nah YH, Yazaki E, Evans DF. The role of gastrointestinal endoscopy in long-distance runners with gastrointestinal symptoms. Eur J Gastroenterol Hepatol. 2001;13(9):1089-1094.

11. Lucas W, Schroy PC, 3rd. Reversible ischemic colitis in a high endurance athlete. Am J Gastroenterol. 1998;93(11):2231-2234.

12. Cohen DC, Winstanley A, Engledow A, Windsor AC, Skipworth JR. Marathon-induced ischemic colitis: why 
running is not always good for you. Am J Emerg Med. 2009;27(2):255 e255-257.

13. Moses FM, Brewer TG, Peura DA. Running-associated proximal hemorrhagic colitis. Ann Intern Med. 1988;108(3):385-386.

14. Heer M, Repond F, Hany A, Sulser H, Kehl O, Jager K. Acute ischaemic colitis in a female long distance runner. Gut. 1987;28(7):896-899.

15. Clausen JP. Effect of physical training on cardiovascular adjustments to exercise in man. Physiol Rev. 1977;57(4):779-815.

16. Carratu R, Parisi P, Agozzino A. Segmental ischemic colitis associated with nonsteroidal antiinflammatory drugs. J Clin Gastroenterol. 1993;16(1):31-34.
17. Deana DG, Dean PJ. Reversible ischemic colitis in young women. Association with oral contraceptive use. Am J Surg Pathol. 1995;19(4):454-462.

18. Wang YF, Zhang H, Ouyang Q. Clinical manifestations of inflammatory bowel disease: East and West differences. J Dig Dis. 2007;8(3):121-127.

19. Silverstein MD, Loftus EV, Sandborn WJ, Tremaine WJ, Feagan BG, Nietert PJ, Harmsen WS, et al. Clinical course and costs of care for Crohn's disease: Markov model analysis of a population-based cohort. Gastroenterology. 1999;117(1):49-57.

20. Road Running Information Center (RRIC) Annual Marathon Report 2009. Available at: http://www.runningusa.org/node/16414\#16589 . Accessed August 4, 2010. 